\title{
Jeder lacht für sich allein
}

\section{Zum Unterschied von Interaktion unter Anwesenden und un- ter Abwesenden}

| STEFAN KÜHL | G

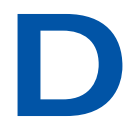

ie Ersetzung der Präsenzlehre durch Fernunterricht aufgrund der Corona-Pandemie hat unter Lehrenden zu sehr unterschiedlichen Einschätzungen geführt. Für die einen stellt die kurzfristige Umstellung auf die Fernlehre eine Zumutung dar, weil weder die technischen noch didaktischen Voraussetzungen dafür beständen. Einzige Konsequenz könne, so die Schlussfolgerung, die staatliche Verordnung eines „Nichtsemesters" sein, in dem Dozenten und Studierende vom Druck des Lehrens und Lernens befreit wer-

»In internetbasierten Interaktionen spürt man nicht die Spannung einer interessanten Diskussion. « den. Andere sehen im erzwungenen Ausfallen der Präsenzlehren die Möglichkeit, der webbasierten Lehre einen entscheidenden Schub zu versetzten. Teilweise herrscht bei technikaffinen Lehrenden eine wahre Euphorie, weil sie sich erhoffen, endlich die Konzepte einer internetbasierten Lehre umsetzen zu können, die durch das bisher übliche routinemäßige Abspielen von zweistündigen Präsenzveranstaltungen blockiert worden ist.

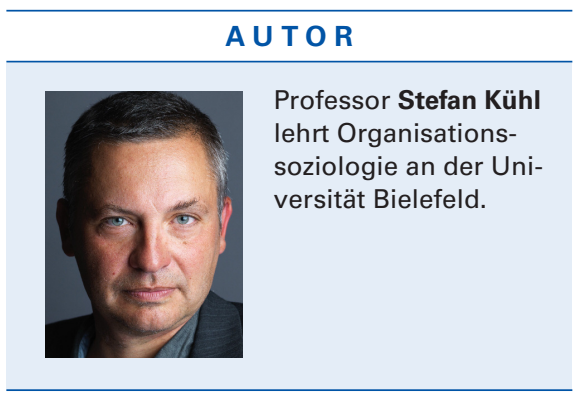

Für Interaktionssoziologen ist diese hochschulpolitische Debatte begrenzt interessant. Ihr Forschungsfokus richtet sich darauf, wie sich Interaktion unter Anwesenden - also der Interaktion an einem Ort - von der der Interaktion unter Abwesenden - also der internetbasierten Interaktion - unterscheidet. Beide Formen von Interaktion basieren auf der erfolgreichen wechselseitigen akustischen und visuellen Wahrnehmung der Kommunikationspartner. Die Bandbreite von sprachlichen Verständigungen, von paraverbalen, also nicht sprachlich gefassten Lauten wie Stöhnen, Kichern oder Lachen, sowie von nonverbalen Zeichen wie Mimik, Gestik oder Körperhaltung unterscheiden sich jedoch erheblich.

Auf den ersten Blick fällt auf, wie weitgehend sich die für die Interaktion unter Anwesenden üblichen Mechanismen inzwischen über Plattformen wie GoToMeeting, Google Duo, Jabber, Jitsi Meet, TeamViewer oder Zoom simulieren lassen. Während vor einigen Jahrzehnten die Kommunikation unter Abwesenden bestenfalls über auf verbale und paraverbale Zeichen beschränkte sprachliche Kommunikation zwischen zwei Teilnehmern via Telefon oder Funk bestand, lassen sich heutzutage in

webbasierten Interaktionen Dutzende von Personen audiovisuell zusammenschalten. Man kann über die Plattformen nicht nur die Stimmen der Interaktionsteilnehmer hören, sondern auch ihre Gesichter und häufig sogar einen Teil ihrer Körper sehen. Man kann Aufmerksamkeit fokussieren, in dem man alle auf einen Bildschirm mit einer Präsentation, einem Bild oder einem Film schauen lässt, die Diskussion für alle mitvisualisiert oder alle gleichzeitig an einem Dokument arbeiten lässt. Man kann Kleingruppeninteraktionen initiieren, zwischen diesen hin- und herwandern und über die Chatfunktion oder über parallellaufende Kommunikationsplattformen Nebengespräche führen.

Aber trotz dieser Möglichkeiten filtert die internetbasierte Interaktion immer noch

eine Vielzahl der für die Kommunikation unter Anwesenden typischen Zeichen heraus. Deswegen spürt man in internetbasierten Interaktionen nicht die Spannung einer interessanten Diskussion. Der Ausdruck persönlicher Achtung für einen klugen Gedanken über ein virtuelles Sternchen oder Herzchen in der internetbasierten Interaktion ist im Vergleich zum anerkennenden Nicken in der Interaktion unter Anwesenden grob. Der Scherz eignet sich in der internetbasierten Interaktion unter Abwesenden nicht besonders gut zur Entspannung der Interaktion. Ein auflockernder Witz führt selten $\mathrm{zu}$ einer gefühlsmäßigen Ansteckung der Interaktionsteilnehme. In der internetbasierten Interaktion lacht jeder mehr oder minder für sich allein.

Man kann diesen Mangel an Ausdrucksmöglichkeiten mit guten Grün- 
den beklagen. Gerade in geselligen Interaktionen, die für Kommunikationen außerhalb von Organisationen typisch sind, werden die negativen Effekte dieser Begrenzungen deutlich. $\mathrm{Zu}$ zweit mag man sich über das Telefon, über Facetime oder über Skype ,festquatschen", von größer ausufernden internetbasierten Feiern über Internetplattformen wie Google Hangouts ist nichts bekannt. Für die ungesellige Interaktion, die für die Kommunikation in Organisationen typisch ist, kann jedoch der Mangel an Ausdrucksformen durchaus positive Effekte haben. Gerade in Interaktionen in Organisationen können, darauf haben vor Kurzem Kai Matthiesen und Jonas Spengler hingewiesen, überflüssige Zeichen die Verständigung erschweren. Die Gefahr sei, dass in der Interaktion unter Anwesenden zu vieles aneinander wahrgenommen wird, was für das Diskussionsthema nicht relevant ist, sodass keine oder zumindest zu wenig Aufmerksamkeit auf das Wesentliche gelegt werden könnte.

Das Fehlen paraverbaler und nonverbaler Zeichen ermöglicht in der Interaktion unter Abwesenden eine Fokussierung auf die Sachdimension. Die Rede ist von einer „Büroatmosphäre“, die sich fast zwangsläufig in internetbasierten Interaktionen in Organisationen ausbilden. Diese Fokussierung auf die Sachdimension kann durch eine für alle sichtbare Visualisierung der Dis- kussion noch unterstützt werden. Die Konzentration auf Sachthemen wird allerdings erkauft durch erhebliche Verluste von Informationen in der Sozialdimension. Die Selbstdarstellungsmöglichkeiten als Person sind in der Kommunikation unter Abwesenden stark eingeschränkt. Während das gerade für die gesellige Interaktion außerhalb von Organisationen problematisch ist, kann das für die Interaktion in Organisationen genutzt werden. So wird in Sprechstunden oder Prüfungen die Diskussion von Themen häufig durch die Beschäftigung von Studierenden - und nicht selten auch Lehrenden - mit ihrer Selbstdarstellung überlagert, sodass einiges dafürsprechen könnte, nicht nur in Zeiten organisational angeordneter physischer Distanzierung solche Formate internetbasiert durchzuführen. Der Verlust von Selbstdarstellungsmöglichkeiten führt in der Zeitdimension darüber hinaus noch dazu, dass die Aufmerksamkeitsspannen kürzer werden. Es scheint in der Interaktion unter Abwesenden an entspannender Ablenkung in Form einer kurzen Verständigung mit der Nachbarin, des kurzen Seitenblicks auf einen attraktiven Gesprächspartner oder eines abschweifenden Blicks durch den Raum zu fehlen. Deswegen lassen sich Interaktionen unter Abwesenden häufig nicht genauso lange durchhalten wie Interaktion unter Anwesenden, was die Empfehlungen zu kürzeren Zeitblöcken und rigiderem Zeitregimen in der internetbasierten Interaktion erklären kann.

Die im Alltag üblichen Klagen über instabile Netzverbindungen, limitierte Ausdrucksmöglichkeiten und Probleme in der Beherrschung der Internetdienste verweisen auf die technischen Begrenzungen der Interaktion unter Abwesenden. Soziologisch deutlich interessanter ist, dass die technischen Möglichkeiten in der Interaktion unter Abwesenden teilweise bewusst nicht ausgeschöpft werden, um diese zielgerichtet gestalten zu können. In Zweiergesprächen wird die Videofunktion beispielsweise oftmals ausgeschaltet, um zu verhindern, dass die Teilnehmer zu stark mit ihrer visuellen Selbstdarstellung in der Interaktion beschäftigt sind, in Seminaren die Chat-Funktion deaktiviert, um die Diskussionen für alle sichtbar auf dem Bildschirm visualisieren $\mathrm{zu}$ können. Die technische Filterwirkung in der Interaktion unter Abwesenden ist also nicht nur eine beklagenswerte Limitierung der Ausdrucksmöglichkeiten, sondern bietet ganz im Gegenteil vielfältige Chancen zur Gestaltung der Interaktion in Organisationen.

Eine ausführliche Fassung des Textes mit Literaturangaben findet sich auf www.sozialtheoristen.de 